\title{
Automated discrete element method calibration using genetic and optimization algorithms
}

\author{
Huy Q. Do, Alejandro M. Aragón, and Dingena L. Schott ${ }^{*}$ \\ Faculty of Mechanical, Maritime and Materials Engineering, Delft University of Technology, Mekelweg 2, 2628CD Delft, The \\ Netherlands
}

\begin{abstract}
This research aims at developing a universal methodology for automated calibration of microscopic properties of modelled granular materials. The proposed calibrator can be applied for different experimental set-ups. Two optimization approaches: (1) a genetic algorithm and (2) DIRECT optimization, are used to identify discrete element method input model parameters, e.g., coefficients of sliding and rolling friction. The algorithms are used to minimize the objective function characterized by the discrepancy between the experimental macroscopic properties and the associated numerical results. Two test cases highlight the robustness, stability, and reliability of the two algorithms used for automated discrete element method calibration with different set-ups.
\end{abstract}

\section{Introduction}

Use of the discrete element method (DEM) has been rising within the powder, raw material and bulk material handling industry for analyzing, and designing material handling systems. However, a major barrier to the effective use of DEM for industrial applications is selecting appropriate input parameters so that simulations can accurately reproduce the behavior of real systems. A calibration approach is commonly chosen for the determination of these parameters that are not easily measured in experiment. Calibration using DEM simulations is actually an iterative process of adjusting input parameters such that the macroscopic results of simulations and experiments are equivalent. "Trial and error" empirical procedures are very time-consuming and unpractical, particularly for DEM models involving many parameters being calibrated. Some attempts have been recently made to improve the calibration procedure, e.g., experimental design [1-4], artificial neutral network training [5], and Latin hypercube sampling and Kriging [6]. The readers are referred to [7] for a complete review on calibration. This study aims to improve the DEM calibration procedure using two different optimization methods: genetic algorithms (GAs) and the DIRECT.

\section{Calibration methods}

The flowchart of the calibration method is illustrated in Fig. 1. This iterative process couples the optimization algorithms with DEM modelling. In each iteration, the optimization algorithm uses the objective function values to update the design parameters, e.g., the coefficients of rolling $\left(\mu_{\mathrm{r}}\right)$ and sliding $\left(\mu_{\mathrm{s}}\right)$ friction. The DEM model inputs these parameters to provide the simulated macroscopic result, e.g., the angle of repose $(A o R)$ that is required to compute the objective function. If the predefined stopping criterion has been reached the process terminates, otherwise the next iteration will be performed.

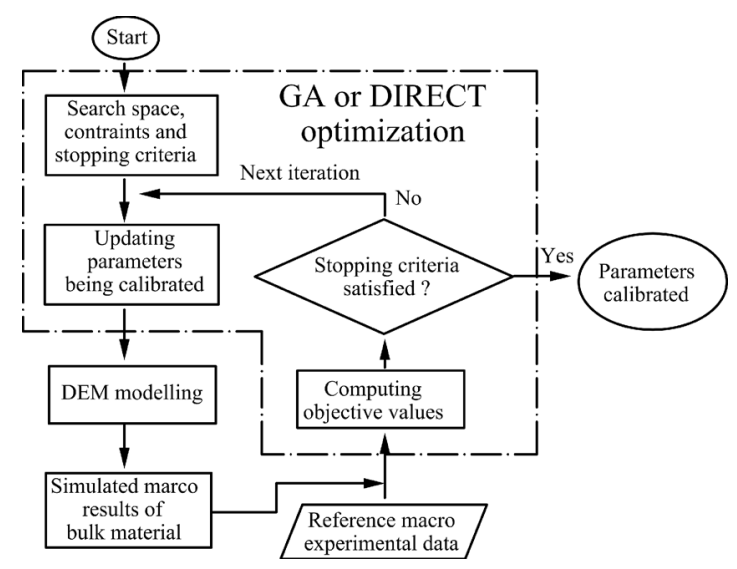

Fig. 1. Flowchart of the calibration process.

\subsection{Genetic algorithms}

Genetic algorithms are search and optimization methods inspired by Darwin's theory of evolution of natural selection and genetics. In GAs, an initial population of candidate solutions evolves through generations (iterations) towards individuals with a better fitness by applying genetic operators such as selection, crossover, and mutation. The fitness function is measured by an objective function of the optimization problem being solved. In each iteration, the better fit individuals are stochastically selected from the current population for breeding to create a new generation. The population is

* Corresponding author: $\underline{\text { d.1.schott@,tudelft.nl }}$

(C) The Authors, published by EDP Sciences. This is an open access article distributed under the terms of the Creative Commons Attribution License 4.0 (http://creativecommons.org/licenses/by/4.0/). 
then updated with the new generation and used for the next iteration of the process. The algorithm terminates when either fitness values of the individuals satisfying a predefined criterion have been found or a predefined maximum number of generations has been obtained or the best fitness value has reached a plateau such that successive iterations no longer produce any improvement.

For further details of GA's theory and applications, the readers are referred to the work of Goldberg [8]. The core components of GA's implementation for our calibration problems are described as the follows:

Initial population and encoding: each calibrated parameter, i.e., $\mu_{\mathrm{r}}$ and $\mu_{\mathrm{s}}$, is binary $\{0,1\}$ encoded and represents a gene. The concatenation of these two genes creates an individual. Initially, a group of individuals is randomly generated and should be seeded on the entire range possible solutions.

Fitness function: defined as the discrepancy between DEM model results and experimental measurement data. Selection: depending on their fitness values, more fit individuals are chosen for crossover.

Crossover: takes chromosomes from selected parents and produces offsprings for the next generation.

Mutation: promotes genetic diversity of the solution set and aims at preventing the GA from converging to a local optimal solution.

\subsection{DIRECT optimization}

Another calibration approach used in this study is DIRECT [9], that is an algorithm for finding the global minimum of a multivariate function subjected to simple bounds without using gradient information. The algorithm was implemented as a subroutine within an open-source library for nonlinear optimization, providing a common interface for several optimization algorithms [10].

DIRECT is a modification of the standard Lipchizian approach, that eliminates the need to specify a Lipschitz constant. The concept of the algorithm is to carry out simultaneous searches on both global and local levels using all possible constants. The DIRECT means "DIviding RECTangles"; this is an iterative process partitioning the search space into a set of hyperrectangles containing a single sampling point at the centre of each rectangle. Initially, the algorithm normalizes the search space by transforming it into the unit hypercube. The objective function is then evaluated at the centre point of this cube. In the first iteration, the unit hypercube is then divided into small hyperrectangles whose centre points are also sampled. In next iterations, all potentially optimal hyper-rectangles are further divided into smaller rectangles whose centre points are sampled. During each iteration, the Graham's scan algorithm is used to find a convex hull that identifies a set of potentially optimal hyper-rectangles. The iterative process continues until either the predefined number of iterations has been reached or a predefined criterion for the objective function or for the variables have been achieved.

\section{DEM simulations and reference results}

To illustrate the calibration approach, LIGGGHTS [11], an open source software, is chosen to conduct the DEM simulations using Hertz-Mindlin contact law with a rolling resistance model [12]. To numerically reproduce the experiments involving hundreds of thousands of sand grains with their actual size, spherical shape is used to reduce the computational complexity. Hourglass and rectangular container simulations on quartz sand grains are carried out to calibrate two main microscopic contact model parameters, i.e., the coefficients of rolling and sliding friction. The rolling friction coefficient of spheres is here introduced to include the shape effect [13]. The experimental measurement data and DEM simulation set-ups of previous work [14] in our research group have been used. Some properties of quartz sand used as the DEM modelling input parameters are listed in Tables 1 and 2. Three macroscopic experimental results are being used here as the references: $A o R$ of $41.57^{\circ}$ and the discharging time of $6.56 \mathrm{~s}$ for the hourglass test, and $A o R$ of $36^{\circ}$ for the rectangular container test. The readers are referred to [14] for further details of these two set-ups.

Two sample test cases for calibrating $\mu_{\mathrm{r}}$ and $\mu_{\mathrm{s}}$ are proposed. In the first sample test case, only the hourglass modelling for $25 \mathrm{~g}$ of sand with the hourglass neck diameter of $5 \mathrm{~mm}$ is used to provide simulated values of $A o R$ and discharging time with varying $\mu_{\mathrm{r}}$ and $\mu_{\mathrm{s}}$. The DEM simulations were conducted at 36 sample points in the $\mu_{\mathrm{r}}-\mu_{\mathrm{s}}$ plane using an evenly spaced regular grid. The contour plots in Fig. 2 and 3 illustrate the AoR and discharging time as functions of the two variables being calibrated, respectively. Compared against the measured values of $A o R$ at $41.57^{\circ}$ and discharging time at $6.56 \mathrm{~s}$, the reference result of the friction coefficients is visualized in Fig. 4, which corresponds to the intersection of the two contour lines.

The second sample test case uses the previous hourglass investigation and another set of 6x6 DEM simulations on the rectangular container to provide simulated values of $A o R$ with varying $\mu_{\mathrm{r}}$ and $\mu_{\mathrm{s}}$. The contour plot in Fig. 5 illustrates the $A o R$ as functions of these two variables. Compared against the measured values of discharging time at $6.56 \mathrm{~s}$ for the hourglass and $A o R$ at $36^{\circ}$ for the rectangular container, the intersection of the two contour lines as shown in Fig. 6 is considered as the reference result.

Table 1. Input particle size distribution of sand for DEM simulations [14].

\begin{tabular}{|c|c|c|c|c|}
\hline Particle diameter $(\mu \mathrm{m})$ & 300 & 425 & 500 & 600 \\
\hline Mass fraction $(\%)$ & 6.21 & 18.29 & 26.05 & 49.45 \\
\hline
\end{tabular}

Table 2. Input properties of sand for DEM simulations [14].

\begin{tabular}{|c|c|}
\hline Particle density $\left(\mathrm{kg} / \mathrm{m}^{3}\right)$ & 2653 \\
\hline Young's modulus $\left(\mathrm{N} / \mathrm{m}^{2}\right)$ & $5 \mathrm{e} 7$ \\
\hline Poisson ratio & 0.3 \\
\hline Coefficient of restitution & 0.9 \\
\hline Coefficient of rolling friction & $0.0-0.5$ \\
\hline Coefficient of sliding friction & $0.2-07$ \\
\hline
\end{tabular}




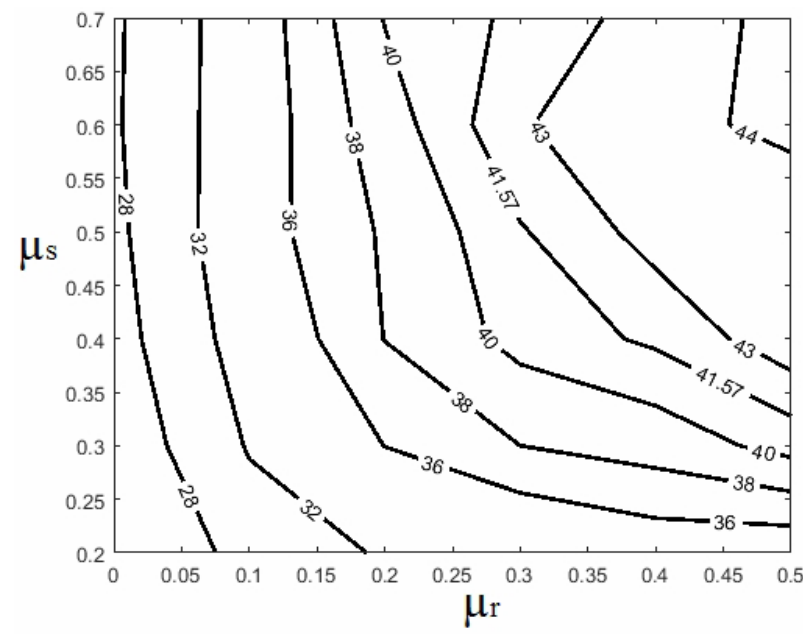

Fig. 2. $A o R$ in degree as a function of the rolling and sliding coefficients from the hourglass simulations [14].

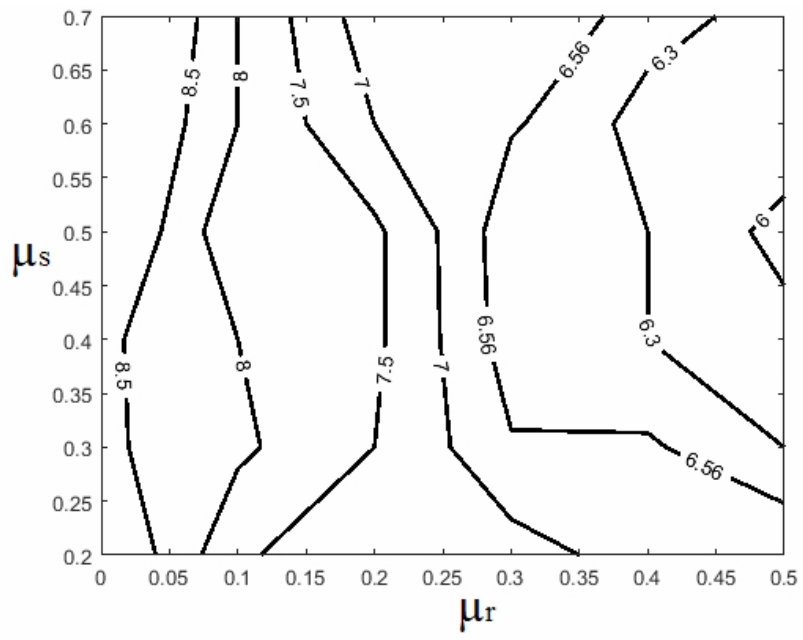

Fig. 3. Discharging time in second as a function of the rolling and sliding coefficients from the hourglass simulations [14].

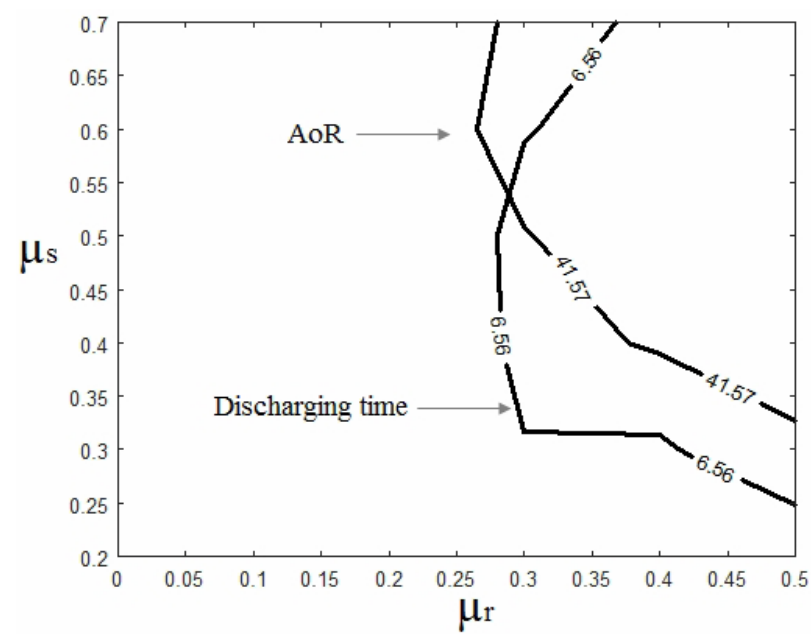

Fig. 4. Reference result defined by the intersection of simulated two contour-lines of $A o R$ and discharging time referred from the experimental results for hourglass [14].

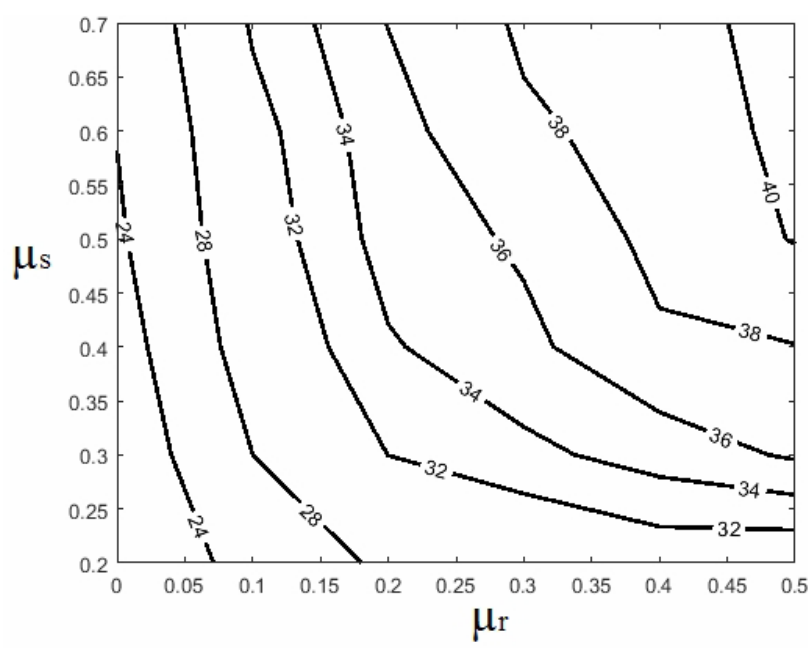

Fig. 5. $A o R$ in degree as a function of the rolling and sliding coefficients from the rectangular container simulations.

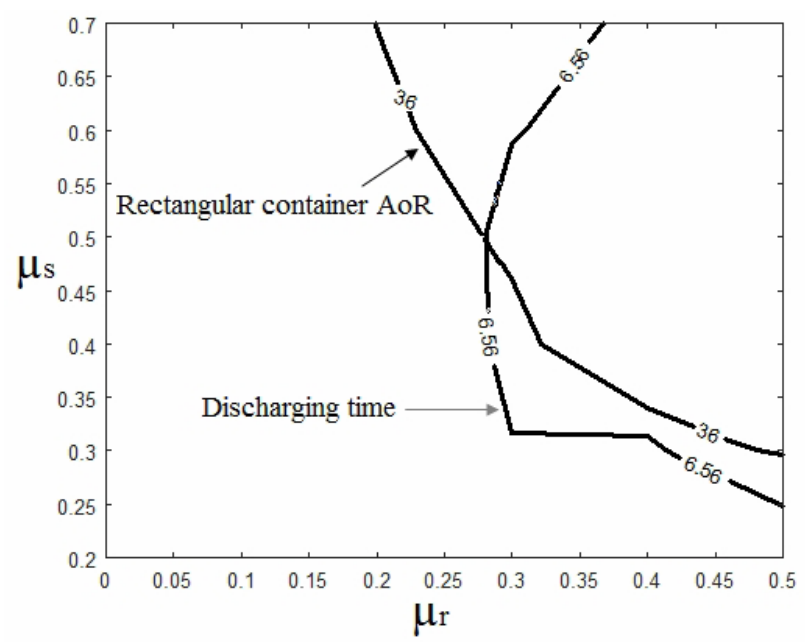

Fig. 6. Reference result defined by the intersection of simulated two contour-lines of hourglass discharging time and rectangular container $A o R$ referred from the experimental results.

\section{Results and Discussion}

DEM simulations are time-consuming. To reduce computational time during the calibration process, the grids of 6x6 sample points in Fig. 2 and 3 are reused for bilinear interpolating values of $A O R$ and discharging time for arbitrary $\mu_{\mathrm{r}}$ and $\mu_{\mathrm{s}}$.

\subsection{Calibration using hourglass - first sample test case}

\subsubsection{Result from $G A$}

Table 3. Input parameters for the GA.

\begin{tabular}{|c|c|}
\hline Population size & 500 \\
\hline Maximum of generation & 30 \\
\hline Chromosome length & 20 \\
\hline Crossover probability & 1.0 \\
\hline Mutation probability & 0.005 \\
\hline
\end{tabular}


Input parameters for the GA are listed in Table 3. A population size of 500 individuals is found to be large enough to be distributed stochastically on the search space. Using its length of 20 bits, a chromosome allocates 10 bits for encoding $\mu_{\mathrm{r}}$ in the range from 0.0 to 0.5 and 10 other bits for encoding $\mu_{\mathrm{s}}$ in the range from 0.2 to 0.7 .

The fitness function $\left(\mathbf{F}_{f i t}\right)$ has been chosen as the total discrepancies on $A o R$ and discharging time (Tdis) between the numerical results and experimental measurement data from the hourglass as:

$$
\mathbf{F}_{\text {fit }}=\left|A o R_{\text {sim }}-41.57\right|+\mid \text { Tdis }_{\text {sim }}-6.56 \mid
$$

100 GA runs are repeatedly performed to search for the possible solutions considering stochastic oscillation. After 30 iterations of all runs, the optimal fitness value has reached a plateau of $0.020 \pm 0.015$, such that successive iterations no longer produce any improvement. The computed rolling and sliding friction coefficients associated with the optimal fitness are $0.284 \pm 0.002$ and $0.543 \pm 0.003$, respectively. The result is in good agreement with the reference result as shown in Fig. 4.

\subsubsection{Result from DIRECT}

The objective function is defined the same way as the fitness function in equation (1). The relative tolerance of the two optimization variables are set to $10^{-3}$ to define a stopping criterion for the algorithm.

Terminating after 259 evaluations, DIRECT outputs the optimal objective value of 0.01 , corresponding to the rolling and sliding friction coefficients of 0.286 and 0.541 , respectively. These results are in good agreement with GA's outcomes in 4.1.1.

\subsection{Calibration using hourglass and rectangular container - second sample test case}

\subsubsection{Result from $G A$}

The fitness function has now been redefined as the total discrepancies between the numerical results and experimental measurement data on discharging time from the hourglass and $A o R$ from the rectangular container as:

$$
\mathbf{F}_{\text {fit }}=\left|\operatorname{Tdis}_{\text {sim } 1}-6.56\right|+\left|A o R_{\text {sim } 2}-36\right|
$$

100 GA runs are carried out targeting the new fitness function. After 30 iterations of all runs, the computed rolling and sliding friction coefficients are $0.277 \pm 0.004$ and $0.502 \pm 0.003$, respectively, and their associated optimal fitness value is $0.007 \pm 0.005$. As expected, the result is in good agreement with the reference result as shown in Fig. 6.

\subsubsection{Result from DIRECT}

The objective function is defined the same way as the fitness function in equation (2). DIRECT has terminated after 275 evolutions. The calibrated values of the coefficients of rolling and sliding friction and their associated optimal value are 0.281, 0.496 and 0.015, respectively; this result is identical to the GA's outcome in 4.1.2.

\section{Conclusions}

This research has demonstrated that the micro properties of quartz sand in DEM model can be automatically calibrated using two methods, i.e., GA and DIRECT optimizations. Both of them are capable of identifying accurately the optimal values within a fairly small number of iterations. The algorithms are robust and stable since they need to evaluate only the fitness or objective function without computing its derivatives. Interpolation grids of the bulk properties resulted from DEM simulations can be incorporated within GA and DIRECT optimizations to reduce the computational cost. The findings of this paper show that the calibration methods can be deployed for a large set of unknown input parameters and other experimental set-ups.

\section{References}

1. J. Yoon, Int. J. Rock. Mech. Min. Sci., 44(6), 871889 (2007)

2. J. Favier, D. Curry, R. LaRoche, Proc. $6^{\text {th }}$ World Congr. Particle. Technol., Nuremberg, Germany (2010)

3. M. Johnstone, Calibration of DEM models for granular materials using bulk physical tests, $\mathrm{PhD}$ thesis, University of Edinburgh, UK (2010)

4. K.J. Hanley, V. O'Sullivan, J.C. Oliveira, K. Cronin, E.P. Byrne, Powder. Technol., 210(3), 230-240 (2011)

5. L. Benvenuti, C. Kloss, S. Pirker, Powder. Technol., 291, 456-465 (2016)

6. M. Rackl, K.J. Hanley, Powder. Technol., 307, 7383 (2017)

7. C.J. Coetzee, Powder. Technol., 310, 104-142 (2017)

8. D.E. Goldberg, Genetic algorithm in search, optimization and machine learning, Adisson-Wesley publishing, Reading Massachusetts, USA (1989)

9. D.R. Jones, C.D. Perttunen, B.E. Stuckman, J. Optim. Theory. Appl., 79(1), 157-181 (1993)

10. http://ab-initio.mit.edu/NLopt

11. C. Kloss, C. Goniva, A. Hager, S. Amberger, S. Pirker, Prog. Comput. Fluid. Dy., 12(2/3), 140-152 (2012)

12. J. Ai, J. F. Chen, J. M. Rotter, J. Y. Ooi, Powder. Technol., 206(3), 269-282 (2011)

13. N. Estrada, E. Azéma, F. Radjai, A. Taboada, Phys. Rev. E, 84(1), 011306 (2011)

14. S. M. Derakhshani, D.L. Schott, G. Lodewijks, Powder. Technol., 269, 127-138 (2015) 